\title{
Period Analyses Without O-C Diagrams
}

\author{
Zdeněk Mikulášek ${ }^{1,2}$, Miloslav Zejda ${ }^{1}$ and Jan Janík ${ }^{1}$ \\ ${ }^{1}$ Department of Theoretical Physics and Astrophysics, Masaryk University, \\ Kotlářská 2, CZ 61137 Brno, Czech Republic \\ email: mikulas@physics.muni.cz \\ ${ }^{2}$ Observatory and Planetarium of J. Palisa, VŠB - Technical University, \\ Ostrava, Czech Republic
}

\begin{abstract}
We present a versatile method appropriate for the period analyses of observations containing phase information of all kinds of periodic or nearly periodic variable stars on the basis of phenomenological modelling of their phase curves and phase functions. The approach is based on rigorous application of a non-linear weighted least-squares method exploiting all available observational data and does not need an O-C diagram as an intermediate stage for period analyses. However, this approach enables us to determine precise times of extrema of light curves, to calculate ephemerides and construct plausible O-C diagrams. We substantiate the general applicability of the method on eclipsing binaries research.
\end{abstract}

Keywords. methods: statistical, binaries: eclipsing, variable stars: other

\section{Introduction}

Most variable stars shows periodic or cyclic changes. Light, spectroscopic or spectropolarimetric changes repeat with one period $P$ or multiple periods. The basic mechanisms of the periodic or nearly periodic stellar variability are the rotation of an anisotropically radiating star, the orbital motions of components in stellar systems, and pulsations or oscillations of various kinds. Basic period analysis consists of finding a reliable ephemeris of the main periodic variation and modelling of the first order effects.

This should be followed by a refined period analysis providing additional pieces of information on the variable star physics, e.g.: 1) Further bodies in the system (stars, planets). 2) Mass exchange between components of interacting binaries. 3) Spin-orbital interactions in the system. 4) Inner stellar structure and general relativity testing by means of the apsidal motion rate. 5) Angular momentum loss through stellar winds. 6) Gravitational waves. 7) Oscillations in the rotation of some chemically peculiar stars.

8) Differential rotation of cool stars.

These period variations are usually delicate; consequently a refined tool is needed for their analysis!

\section{The standard O-C diagram method and its afflictions}

The majority of period analyses were done using O-C diagrams or their modifications. $O$ is the observed time of a light curve (LC) extremum, $C$ is the time of the same extremum calculated by means of an ephemeris (as a rule linear). Historical $O$ times and their uncertainties can be taken from the literature or specialised databases.

The credibility of obtained astrophysical information strictly depends on the reliability of $O$ times and their uncertainties. Extrema of LCs used to be rather badly defined and even small imperfections in observations in the vicinity of the extremum may cause 
large deflections in the individual $O$ determinations. The majority of published times of variable stars' extrema were obtained by means of the notorious Kwee-van Woerden method and its modifications. Unfortunately, the method is often used as a black box in an inappropriate way for data where authors themselves ruled out usage of this method. Furthermore, we know from our experience that this method exploits only a minor part of the information hidden in observations that results in the enlargement of the $O$ scatter by two or three times. Quite unusable is the uncertainty estimate which used to be several times underestimated.

The crucial affliction of $O$ values determined by standard methods is based on the fact that they do not take into account that most variable star changes are repeating. Knowledge of the form of light curves derived from past observations can enhance the credibility of $O$ determinations considerably. Then, we can use the method of the phase shift of an observed light curve (LC) with respect to a template LC which provides more reliable results and estimates of the uncertainties. Unfortunately, such methods are utilised relatively rarely.

Standard O-C diagrams are not a very reliable tool for period analysis since they have their specific limitation. What shall we do to improve the reliability of such demanding period analyses? We can improve their input using reliable $O$ values using the periodicity LC. But even better: We can cancel the O-C intermediate step completely! We can analyse period variations directly using original observations of all kinds!

The need for original observational data (magnitudes) is the only limitation of the method. In several instances, we are unable to obtain the original data, so we have to be satisfied with the published $O$ value.

\section{Method of direct period analyses and modelling of period variations}

The techniques used to analyse the data are based on the rigourous application of the non-linear, weighted, least-squares methods used simultaneously for all relevant data containing phase information. Our technique does not utilise an O-C diagram as an intermediate stage of data processing; O-C diagrams are used only as a visual check on the adequacy of the models. The method can be successfully applied to continuous time series and surveys like ASAS or Hipparcos.

Let us assume that all observed phase curves of a star are well-described by the unique general model function $F(\vartheta, \mathbf{a})$, described here by $g_{a}$ parameters contained in a parameter vector $\mathbf{a}, \mathbf{a}=\left(a_{1}, \ldots, a_{j}, \ldots, a_{g_{a}}\right)$. In our computation, we assume that the form of all the phase variations is constant and the time variability of the observed quantities are given by a phase function $\vartheta(t, \mathbf{b})$, which is a continuous monotonic function of time $t$. The fractional part of it corresponds to the common phase, the integer part of it being the so called epoch $(E)$. We can express the phase function by means of a simple model quantified by $g_{b}$ parameters $\mathbf{b}, \mathbf{b}=\left(b_{1}, \ldots, b_{k}, \ldots, b_{g_{b}}\right)$. The instantaneous period $P(t)$ is connected with the phase function by the following simple relations:

$$
P(t, \mathbf{b})=\left(\frac{\mathrm{d} \vartheta}{\mathrm{d} t}\right)^{-1}, \quad \Rightarrow \quad \vartheta(t, \mathbf{b})=\int_{0}^{t} \frac{d \tau}{P(\tau)} .
$$

For a realistic modeling of phase variations for all data types, we need $g_{a}$ free parameters for the description of the model function $F(\vartheta, \mathbf{a})$, and $g_{b}$ free parameters for the description of the phase function $\vartheta(t, \mathbf{b})$. The computation of the free parameters is iterative under the basic condition that the weighted sum $S(\mathbf{a}, \mathbf{b})$ of the quadrates of the 
difference $\Delta y_{i}$ of the observed value $y_{i}$ and its model prediction is minimal ( $w_{i}$ being the individual weight of the $i$-th measurement).

$$
\begin{gathered}
\Delta y_{i}=y_{i}-F\left(\vartheta_{i}\right) ; \quad S=\sum_{i=1}^{n} \Delta y_{i}^{2} w_{i} ; \quad \delta S=\mathbf{0} ; \quad \Rightarrow \\
\sum_{i=1}^{n} \Delta y_{i} \frac{\partial F\left(\vartheta_{i}, \mathbf{a}\right)}{\partial a_{j}} w_{i}=0 ; \quad \sum_{i=1}^{n} \Delta y_{i} \frac{\partial F}{\partial \vartheta_{i}} \frac{\partial \vartheta\left(t_{i}, \mathbf{b}\right)}{\partial b_{k}} w_{i}=0 .
\end{gathered}
$$

We obtain here $g=g_{a}+g_{b}$ equations of $g$ unknown free parameters. The weights of individual measurements $w_{i}$ are inversely proportional to their expected uncertainty. The system of equations is always non-linear; we have to determine the parameters iteratively. Nevertheless, with a good initial estimate of the parameter vectors $a$ and $b$, the iterations converge very quickly. Usually, we need only several tens of iterations to complete the whole iteration procedure.

\subsection{Application to eclipsing binaries}

Most of the studies dealing with orbital period changes of eclipsing variables are based on the O-C diagram techniques, even when several advanced physical codes for EB light curves solutions as PHOEBE, FOTEL or Wilson-Devinney codes offer direct period analysis in the sense of the above outlined method. We recommend the use of these utilities as you can eventually at least use the simulated light curve of an EB as a template phase curve.

Even more simple is to use the phenomenological models of the light curve. We developed a bundle of EB LC models which are able to describe the majority of real cases more than satisfactorily using a minimum of free parameters. For example, the following phenomenological model can be applied to close eclipsing binaries with zero eccentricity (minima at phases $\varphi=0,0.5$ ). The model satisfactorily well describes the primary and secondary eclipse of various forms (even U shape minima), proximity effects, and O'Connell's effect using only seven free parameters.

$$
\begin{gathered}
y(t) \simeq y_{0}+a_{1}\left\{1-\left\{1-\exp \left[-\left(\varphi_{\mathrm{I}} / d\right)^{2}\right]\right\}^{C}\right\}+a_{2}\left\{1-\left\{1-\exp \left[-\left(\varphi_{\mathrm{II}} / d\right)^{2}\right]\right\}^{C}\right\} \\
+a_{3} \cos (4 \pi \vartheta)+a_{4}\left[\sin (2 \pi \vartheta)-\frac{1}{2} \sin (6 \pi \vartheta)+\frac{1}{10} \sin (10 \pi \vartheta)\right]
\end{gathered}
$$

where

$$
\vartheta=\left(t-M_{0}\right) / P ; \varphi_{\mathrm{I}}=\vartheta-\operatorname{round}(\vartheta) ; \varphi_{\mathrm{II}}=\vartheta-\text { floor }(\vartheta)-\frac{1}{2}
$$

$y_{0}$ is the mean magnitude outside of eclipses, $a_{1}$ and $a_{2}$ are the depths of the primary and the secondary eclipses, $d$ is the parameter describing the width of eclipses, $C$ determines the sharpness of eclipses, $a_{3}$ is the semi-amplitude of changes outside eclipses and $a_{4}$ is the amplitude of the O'Connell effect.

\section{Instead of conclusions - something for O-C diagram lovers}

It would be unreasonable to abandon O-C diagrams completely. The O-C diagram can help us even if we use the method of direct period analyses, namely in the stage of finding adequate models for the the period changes. Fortunately, we can create the classical O-C diagram using so called virtual O-C values for any subsets of observational data. In addition, we can easily construct a diagram illustrating changes of the instant period $P(t)$.

Using the residuals of the observed data $\Delta y_{i}$, we can create individual values of the phase shifts expressed in days $(\mathrm{O}-\mathrm{C})_{j}$ with adapted individual weight $W_{j}$ for each observed datum, and averages of the phase shifts defined for arbitrarily selected groups of 
measurements ${\overline{(\mathrm{O}-\mathrm{C})_{i}}}_{\text {, }}$ or deflection of the mean period from the instant model period $\Delta P_{k}\left(t_{k}\right)$ :

$$
\begin{gathered}
(\mathrm{O}-\mathrm{C})_{j}=-P\left(t_{j}\right) \Delta y_{j}\left(\frac{\partial F}{\partial \vartheta}\right)^{-1} ; \quad W_{j}=\left(\frac{\partial F}{\partial \vartheta}\right)^{2} w_{j} \\
{\overline{(\mathrm{O}-\mathrm{C})_{k}}}_{k}=\frac{\sum_{j=1}^{n_{k}}(\mathrm{O}-\mathrm{C})_{j} W_{j}}{\sum_{j=1}^{n_{k}} W_{j}} ; \quad \Delta P_{k}=\frac{\sum_{j=1}^{n_{k}}(\mathrm{O}-\mathrm{C})_{j} \vartheta_{j} W_{j}}{\sum_{j=1}^{n_{k}} \vartheta_{j}^{2} W_{j}} .
\end{gathered}
$$

Computations of ${\overline{(\mathrm{O}-\mathrm{C})_{k}}}_{k}$ and $\Delta P_{k}$ follow after finding of the model parameters; consequently, they have no influence on the model solution. They are used only to visualise the solution. Similarly, we can compute virtual 'observed' values of the instant period from a group of observations to generate the model curves in our figures.

\section{Acknowledgements}

This work was supported by the grants GAAV IAA 301630901, GAČR 205/08/0003, MEB 0810095, and MUNI/A/0968/2009. The authors thank S. N. de Villiers for his kind improvement of the manuscript language.

\section{Discussion}

C. Chambliss: O-C diagrams are sometimes misused. Sinusoidal O-C diagrams have been used in some cases to infer the existence of third light components that later have proven not to exist. [AK Herculis is an example.] Also how good are old photographic or visual estimates?

Z. MiKulÁŠEK: In general, visual estimates of eclipsing binary minima timings are not a very reliable source of astrophysical information because of the subjective character of observation and processing. The credibility of visual observations could be enhanced if we treat the complete time series of visual estimates of brightness. Such observations could be processed by the method of direct period analysis. Unfortunately, we generally only have estimates of times of minima at our disposal, not original observation data.

On the contrary, time series of photographic surveys used to be a relatively solid source, namely if we have at our disposal several hundreds of magnitudes individually derived from photographic plates. Such material is optimal for direct period analysis. Times of minima determined from several measurements are relatively reliable. However, we must be suspicious in the case of so called 'weakenings' which are not the time of the photographic minimum but only information that the star is dimmer than usual at a given moment. Such estimates of times of minima used to be even worse sources of information than visual minima time estimates.

R. WILSON: I like this scheme very much. The procedure has been in the WD program for about 12 years, and has been applied in several papers by myself and by others. As formulated in WD, it can supply the usual ephemeris parameters (reference time, period $(\mathrm{P})$, and $\mathrm{dP} / \mathrm{dt})$ in combination with apsidal motion $(\mathrm{d} \omega / \mathrm{dt})$, third body parameters, and all other EB parameters. It can be used with mixed light and velocity curves so as to fill gaps in coverage.

Z. MikulášEK: We thank you for this note; we hope that our paper will contribute so that period analyses will be done more properly. 